\title{
Power Control and Utility Optimization in Wireless Communication Systems
}

\author{
Dimitrie C. Popescu and Anthony T. Chronopoulos \\ Electrical Engineering Dept. Computer Science Dept. \\ University of Texas at San Antonio \\ 6900 N Loop 1604 W, San Antonio, TX 78249 \\ Email: dimitrie.popescu@utsa.edu, atc@cs.utsa.edu
}

\begin{abstract}
In this paper we present an analysis of power control algorithms established over the past decade for cellular telephone systems, in conjunction with utility functions introduced recently to specify quality of service in wireless systems providing data services. These algorithms are compared with power control algorithms based on game theory established relatively recently. The analysis shows that the Nash equilibrium points to which the game theory based algorithms for power control converge are not efficient, and that better solutions are possible.
\end{abstract}

\section{INTRODUCTION}

Transmitter power control is a main component of radio resource management at the physical layer of a wireless communication system, and contributes to minimizing interference and increasing system capacity. Transmitter power control contributes also to extending the battery life of mobile terminals by ensuring that these transmit at the minimum power level necessary to achieve a specified quality of service (QoS). Power control is extremely important in Code Division Multiple Access (CDMA) systems which have received increasing attention lately as a multiple access scheme for future generation wireless systems. CDMA systems are interference limited, and power control is an effective way of reducing the multiple access interference (MAI) and maintaining the specified QoS requirements.

QoS can be defined in terms of a minimum signal-tointerference-ratio (SIR) or signal-to-interference-plus-noiseratio (SINR) $\gamma_{k}^{*}$ for a given mobile terminal $k$, and the QoS requirement may be expressed as

$$
\gamma_{k} \geq \gamma_{k}^{*} \quad \forall k
$$

The value $\gamma_{k}^{*}$ is a threshold which ensures transmission quality for user $k$, and may be determined from the bit error rate (BER) requirement that corresponds to the service performed by mobile terminal $k$. This threshold-type definition for QoS is well suited for terminals performing voice services like cellular telephones, for which providing acceptable speech quality at the telephone receiver is directly related to the SINR of the mobile terminal [3]. In this case the target SIR is the same for all users in the system, $\gamma_{k}^{*}=\gamma^{*}, \forall k$ and depends on the desired quality of speech at the receiver phone. We note that numerous power control algorithms based on ensuring a QoS criterion as specified by equation (1) have been developed for cellular telephone systems providing voice services over the past decade [2], [4], [6], [9], [11], [13].

When the mobile terminal performs data transmission services, as it is the case in wireless networks, the above QoS definition is no longer appropriate [3], and concepts from microeconomics have been used lately to define QoS in wireless systems in terms of utility functions. In general, the utility function measures the satisfaction of a given user with a specific service, and in wireless communication systems utility can be related to the SIR. From this perspective different services, like voice and data, will have QoS described by different utility functions. Based on this new formulation for QoS, new power control algorithms for wireless systems have been developed recently using a game theoretic approach to maximize the utility functions [3], [8], [12].

In our paper we investigate the use of power control algorithms established for voice systems [2], [7], [9], [11] in conjunction with utility functions used to specify QoS for data services [3], [8], [12]. Our work is motivated by the fact that these algorithms are simple and well understood, and are already present in current cellular telephone systems, thus offering an alternative to the implementation of newly developed algorithms in initial deployment of future generation wireless systems. We note that, while not specifically designed for utility maximization, these algorithms provide comparable performance in terms of the value of utility functions to the recently established utility maximizing algorithms for power control [8], [12], thus allowing a smooth transition to new implementations for next generation systems.

\section{UTILITY FUNCTIONS FOR WIRELESS SySTEMS}

The concept of utility is commonly used in microeconomics and game theory to denote the level of satisfaction of a decision-maker with specific products or services as a result of its actions [10, Ch. 2]. In wireless communication systems the level of satisfaction is related to the QoS that a mobile terminal receives which is typically expressed in terms of BER or SIR requirements.

In cellular telephone systems providing voice services, utility is a step-like function of the SIR [3] which matches the threshold QoS requirements in equation (1) that correspond to 
voice services:

$$
u_{k}=\left\{\begin{array}{cl}
0 & \text { for } \quad \gamma_{k}<\gamma_{k}^{*} \\
\mathcal{U}_{k} & \text { for } \quad \gamma_{k} \geq \gamma_{k}^{*}
\end{array} \quad \forall k\right.
$$

where $\mathcal{U}_{k}$ is the value of the utility function for user $k$.

Utility functions for wireless systems providing data services depend usually on both the SIR and the transmitted power of a given terminal. This is because power is a valuable commodity for a mobile terminal, and transmitting at the lowest possible power will extend the terminal's battery life and contribute to increasing its level of satisfaction. One choice of utility function proposed in [3] assumes that mobile terminals transmit data in frames (or packets) of length $M$ bits, containing $L<M$ information bits at a data rate of $R$ bits/s, and expresses the utility function for terminal $k$ with transmit power $p_{k}$ Watts as

$$
u_{k}=\frac{\operatorname{LRf}\left(\gamma_{k}\right)}{M p_{k}} \quad \forall k
$$

where

$$
f\left(\gamma_{k}\right)=\left(1-2 P_{e_{k}}\right)^{M}
$$

is the efficiency function for terminal $k$ with $P_{e_{k}}$ being the terminal's BER. In this case the utility function depends explicitly on the terminal's transmit power $p_{k}$, and implicitly on the terminal's SIR $\gamma_{k}$. The dependence on the SIR $\gamma_{k}$ is through $P_{e_{k}}$ which depends on $\gamma_{k}$ and the particular modulation scheme employed. For example for BPSK modulation $P_{e_{k}}=Q\left(\sqrt{2 \gamma_{k}}\right)$, for DPSK modulation $P_{e_{k}}=0.5 e^{-\gamma_{k}}$, for coherent FSK $P_{e_{k}}=Q\left(\sqrt{\gamma_{k}}\right)$, and for non-coherent FSK $P_{e_{k}}=0.5 e^{-0.5 \gamma_{k}}$. The utility function in equation (3) is measured in bits/Joule and has a nice physical interpretation as the number of information bits received successfully per Joule of energy consumed for transmission. We note that, a linear pricing factor of the form $a_{k} p_{k}$ may be included to improve the efficiency of the power control algorithms based on maximizing the utility function in equation (3) as it was done in [12].

An alternative to this function considers the channel capacity as the figure of merit and uses it to derive power control algorithms [8]. To provide a distributed implementation, capacity is approximated by the Gaussian channel capacity formula, and the utility function is expressed as

$$
u_{k}=R \log _{2}\left(1+\gamma_{k}\right)-a_{k} p_{k} \quad \forall k
$$

where $W$ is the available bandwidth for communication, and $a_{k}$ is the pricing factor that corresponds to user $k$. The term $a_{k} p_{k}$ is a linear cost on transmit power and is included to ensure that the utility maximization problem is well-defined.

\section{Distributed Power Control Algorithms}

Algorithms for power control in wireless systems can be centralized or distributed. We note that centralized power control (CPC) algorithms require a central controller which knows the parameters of all radio links in the wireless system, and are therefore not easy to be implemented [5]. In contrast, in distributed power control (DPC) algorithms only knowledge of a given terminal's link is needed in order to adjust its transmitted power independent of the other terminals. As a consequence DPC algorithms have lower complexity and require less computational power than CPC algorithms, and are preferred in practical implementations. In this paper we consider DPC algorithms based on ensuring a QoS criterion as specified by equation (1), as well as algorithms based on maximizing utility functions in equations (3) and (5).

We consider the uplink of a single-cell of a CDMA wireless system with $K$ mobile terminals transmitting data to the base station. Each terminal transmits $L$ information bits in frames (or packets) of length $M$ bits at a fixed rate of $R$ bits/sec. We denote the $j^{\text {th }}$ terminal transmitted power by $p_{j}$, $j=1,2, \ldots, N$, and the path gain of terminal $j$ to the base station by $h_{j}, j=1,2, \ldots, K$. The signal to interference ratio corresponding to terminal $j$ is given by [12]

$$
\gamma_{j}=\frac{W}{R} \frac{h_{j} p_{j}}{\sum_{k=1, k \neq j}^{N} h_{k} p_{k}+\sigma^{2}}
$$

where $W$ is the available bandwidth expressed in $[\mathrm{Hz}]$, and $\sigma^{2}$ is the average power of additive Gaussian white noise (AWGN) power at the receiver. This expression assumes that users in the CDMA system are assigned pseudorandom noise (PN) sequences, and that conventional matched filter detectors are used at the receiver [12]. We note that the SIR expression for CDMA systems in equation (6) is similar to the SIR expressions used in early papers on power control for cellular radio systems providing voice services [2], [4], [7] which assumed a system with a finite set of channels (either time or frequency slots) rather than a CDMA scheme. We also note that the SIR expression in equation (6) was also used in more recent DPC algorithms [11].

A very simple DPC algorithm adjusts the transmitted power of mobile terminals independently at discrete time instances by increasing the transmitted power for a given terminal $k$ if the corresponding SIR $\gamma_{k}$ is below the specified target $\gamma_{k}^{*}$, or decreasing the transmitted power if the corresponding SIR $\gamma_{k}$ is above the specified target $\gamma_{k}^{*}$. The associated power update equation is given by [2]

$$
p_{k}^{(n)}=p_{k}^{(n-1)} \frac{\gamma_{k}^{(n-1)}}{\gamma_{k}^{*}}, \quad 1 \leq k \leq K, n \geq 1
$$

When the specified target SIRs $\gamma_{k}^{*}$ are feasible then the DPC algorithm converges to a fixed point where equation (1) is satisfied [2], [13]. Otherwise, when the target SIRs are not feasible, transmit powers keep increasing indefinitely in an attempt to reach the specified SIR values, and the DPC algorithm will not converge. To avoid this situation, the DPC algorithm described by equation (7) is modified, and an upper bound on transmit power is imposed. The power update equation becomes then

$$
p_{k}^{(n)}=\min \left\{P_{\max }, p_{k}^{(n-1)} \frac{\gamma_{k}^{(n-1)}}{\gamma_{k}^{*}}\right\}, 1 \leq k \leq K, n \geq 1
$$


and corresponds to the constrained DPC (CDPC) algorithm [7]. For feasible target SIRs the CDPC algorithm converges to the fixed point where where equation (1) is satisfied for all terminals. When the target SIRs are not feasible the CDPC will reach a fixed point where equation (1) is satisfied for only a subset of terminals, while the other terminals for which equation (1) is not satisfied will transmit at the maximum allowed power level.

The DPC and CDPC algorithms are first-order algorithms in the sense that they require only the current power level in the power update equation. For faster convergence a secondorder power control (SOPC) algorithm was proposed [9]. This requires power levels at current and previous iterations, and the associated power update equation is

$$
\begin{aligned}
p_{k}^{(n)}= & \min \left\{P_{\max }, \max \left\{P_{\min }, \omega^{(n)} p_{k}^{(n-1)} \frac{\gamma_{k}^{(n-1)}}{\gamma_{k}^{*}}+\right.\right. \\
& \left.\left.+\left[1-\omega^{(n)}\right] p_{k}^{(n-2)}\right\}\right\}, 1 \leq k \leq K, n \geq 1
\end{aligned}
$$

where $\omega$ is the relaxation factor which may be fixed or may vary according to the iteration [9], and $P_{\min }$ is a lower bound on transmit power imposed to avoid non-positive values for power. We note that, when $\omega$ is fixed and equal to 1 , the power update in equation (9) becomes identical to that in equation (8), and the SOPC algorithm reduces to the CDPC algorithm.

Recently, an alternative to the CDPC algorithm described by equation (8) was proposed [11]. The exponential DPC algorithm (EDPC) uses an exponential function of the SIR, and the associated power update equation is given by [11]

$p_{k}^{(n)}=\min \left\{P_{\max }, p_{k}^{(n-1)} e^{\xi\left[\gamma_{k}^{*}-\gamma_{k}^{(n-1)}\right]}\right\}, 1 \leq k \leq K, n \geq 1$

where $\xi>0$ is a parameter which should be optimized for fast convergence speed. We note that the power update in equation (10) can be rewritten as

$$
p_{k}^{(n)}=\min \left\{P_{\max }, p_{k}^{(n-1)} \frac{e^{-\xi \gamma_{k}^{(n-1)}}}{e^{-\xi \gamma_{k}^{*}}}\right\}, 1 \leq j \leq N, n \geq 1
$$

which shows that in this case the power update equation is similar to that of previous DPC algorithms, but uses an exponential function of the SIR rather than the SIR directly. The motivation for this choice is based on the convergence properties of the exponential function [11] which converges asymptotically when time approaches infinity, and for which the convergence speed may be adjusted through the positive parameter $\xi$.

We note that both the SOPC and EDPC algorithms are power constrained, and that they behave similar to the CDPC with respect to the target SIRs: when these are feasible they will converge to a point where equation (1) is satisfied for all terminals, otherwise at the fixed point equation (1) is satisfied for only a subset of terminals, while the other terminals will transmit at the maximum allowed power level.
Using a game theoretic approach distributed power control is formulated as a non-cooperative game in [12] in which users adjust transmit powers to maximize their corresponding utility functions in equation (3). The resulting utility maximization algorithm (UTI1) has a power update similar to that in equation (8) with the difference that the users update powers only if their corresponding utility is not decreased by the power update, otherwise they keep their powers unchanged. The value of $\gamma_{k}^{*}$ in this case is the SIR that corresponds to a Nash equilibrium point of the non-cooperative power control game and will be discussed in Section IV.

An alternative game theoretic approach is presented in [8], and uses the utility functions in equation (5). The resulting distributed power control algorithm (UTI2) has the following power update equation

$$
\mathbf{p}^{(n)}=F\left(\mathbf{M} \mathbf{p}^{(n-1)}+\mathbf{b}\right) \quad n \geq 1
$$

where $\mathbf{p}$ is the vector containing user powers, $\mathbf{b}$ is a constant $K$-dimensional vector with elements

$$
b_{k}=\frac{R}{a_{k} \ln 2}-\frac{R \sigma^{2}}{W h_{k}}
$$

$\mathbf{M}$ is a $K \times K$ matrix expressed in terms of user path gains as

$$
\mathbf{M}=\frac{R}{W}(\mathbf{I}-\mathbf{H})
$$

with $\mathbf{I}$ being the identity matrix of order $K$, and elements of matrix $\mathbf{H}$ being $h_{i j}=h_{i} / h_{j}$ (with $h_{i i}=1$ ), and $F(\cdot)$ is a diagonal mapping from $\mathbb{R}^{K}$ to $\mathbb{R}^{K}$ given by

$$
F_{k}(x)=\left\{\begin{array}{lll}
P_{\max } & \text { if } \quad x>P_{\max } \\
x & \text { if } \quad 0 \leq x \leq P_{\max } \\
0 & \text { if } \quad x<0
\end{array}\right.
$$

No target/equilibrium SIRs are used in the power update in this case, and the resulting equilibrium SIRs depend on the pricing strategy used [8].

\section{TARget SIRS AND UTILITY MAXIMIZATION}

In general, in cellular telephone systems users have uniform QoS requirements, which imply uniform target SIRs that depend on the desired quality of the speech signal at the receiver phone. This is a subjective QoS measure and has no direct relationship to system operating parameters like link quality or the type of modulation used for transmission. We note that power control algorithms established for voice systems presented in Section III (DPC, CDPC, SOPC, EDPC) work also with non-uniform SIRs, as long as these are feasible. When the set of non-uniform specified SIRs are not feasible and no maximum power limits are set, user powers increase indefinitely in an attempt to meet the specified targets. In the case of constrained power control algorithms (like the CDPC, SOPC, or EDPC), with maximum power limits and unfeasible SIRs, the algorithms stop when the maximum power value for all users is reached, regardless of the resulting SIR values. When only a subset of the specified non-uniform SIRs is feasible constrained power control algorithms converge to a fixed point where equation (1) is satisfied for only a subset 
of terminals, while the other terminals for which equation (1) is not satisfied will transmit at the maximum allowed power level.

In power control algorithms based on utility maximization, UTI1 [12] and UTI2 [8], user SIRs at the end of the algorithm are not established a priori as in the case of DPC, SOPC, or EDPC algorithms, but rather correspond to a Nash equilibrium point for the system. At a Nash equilibrium point no terminal can further increase its corresponding utility through individual action. User SIRs at a Nash equilibrium point depend on actual characteristics of the wireless system like the link quality or modulation scheme employed. For the UTI1 power control algorithm the unique Nash equilibrium point of the corresponding non-cooperative power control game is obtained from the following relation [12]

$$
f\left(\gamma_{k}^{*}\right)=f^{\prime}\left(\gamma_{k}^{*}\right) \gamma_{k}^{*}
$$

where $f^{\prime}(\cdot)$ is the first derivative of the efficiency function $f(\cdot)$. We note that different modulation schemes have different efficiency functions and imply in general different equilibrium SIRs, and that even for the same modulation scheme different packet lengths $M$ imply different equilibrium SIRs [1].

For the UTI2 power control algorithm different pricing strategies implied by the user pricing factor $a_{k}$ imply different equilibrium SIRs [8]. We note that in this case a fair allocation of SIRs is obtained when the pricing factor $a_{k}$ for a given user $k$ is proportional to the corresponding path gain $h_{k}$, and results in uniform SIRs for all users in the system [8].

To conclude this section we note that, if the SIRs corresponding to Nash equilibrium points of non-cooperative power control games are feasible for DPC, SOPC, or EDPC algorithms, then one could use these algorithms as an alternative way of reaching the equilibrium SIRs. However, reaching the equilibrium SIRs does not guarantee that corresponding utility functions will also be maximized, since final powers yielded by different power control algorithms are usually different.

\section{Simulation Results}

We have simulated the power control algorithms presented in Section III for a CDMA system with a single cell and $K=9$ users situated at distances $310 \mathrm{~m}, 460 \mathrm{~m}, 570 \mathrm{~m}, 660 \mathrm{~m}, 740 \mathrm{~m}$, $810 \mathrm{~m}, 880 \mathrm{~m}, 940 \mathrm{~m}$, and $1000 \mathrm{~m}$ from the base station. The available bandwidth is $W=1 \mathrm{MHz}$, the data rate is $R=$ $10 \mathrm{kbps}$, the power spectral density $\sigma^{2}=10^{-15} \mathrm{~W} / \mathrm{Hz}$. For DPC, CDPC, SOPC, and EDPC algorithms the target SIR was identical for all terminals $\gamma^{*}=12.4$. This value was chosen to be equal to the Nash equilibrium SIR for the non-cooperative power control game that define algorithms UTI1 and UTI2, in order to allow a meaningful comparison of considered power control algorithms. We note that for algorithm UTI1 the Nash equilibrium SIR of $\gamma^{*}=12.4$ corresponds to a system using non-coherent FSK modulation that transmits frames of length $M=80$ bits, with $L=64$ information bits per frame, and for algorithm UTI2 uniform equilibrium SIR $\gamma^{*}=12.4$ is obtained for a pricing factor of $a_{k}=1.725 \times 10^{17} h_{k}$.

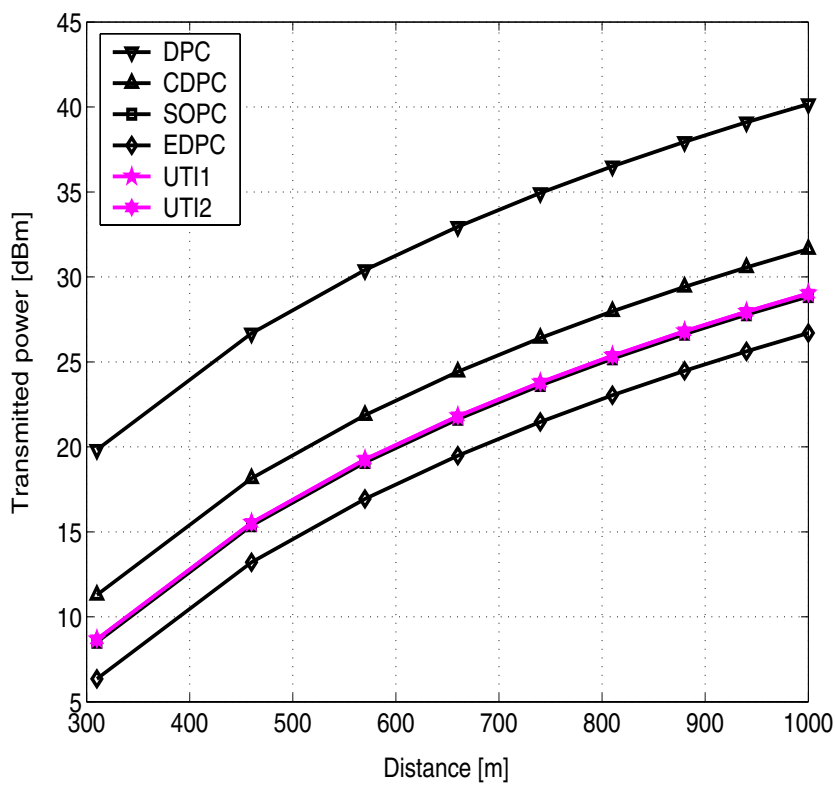

Fig. 1. Final user transmit powers for a system with 9 users after running various distributed power control algorithms discussed in section III.

For the power constrained algorithms (CDPC, SOPC, EDPC, UTI1, and UTI2) the maximum transmit power level is $33.0103 \mathrm{dBm}(2 \mathrm{~W})$ for all terminals. Each algorithm was initialized with the same set of randomly generated transmit powers which was equal to $31.6141 \mathrm{dBm}, 28.64 \mathrm{dBm}$, $30.4409 \mathrm{dBm}, 29.9387 \mathrm{dBm}, 31.4342 \mathrm{dBm}, 31.0109 \mathrm{dBm}$, $29.8067 \mathrm{dBm}, 27.1475 \mathrm{dBm}$, and $31.2104 \mathrm{dBm}$, and the final transmit powers corresponding to all terminals yielded by the algorithms are presented in Figure 1. We note that the target SIR is achieved with minimum transmit power for the EDPC algorithm, and that the unconstrained DPC algorithm implies the maximum transmit power for the same target SIR and initial power values. We also note that the UTI1 and UTI2 algorithms yield transmit powers similar to those obtained with the SOPC algorithm.

The values of the utility function in equation (3) that correspond to the transmit powers yielded by the DPC, CDPC, SOPC, and EDPC algorithms are plotted together with the value of the utility function yielded by algorithm UTI1 in Figure 2. As it is expected from the expression of the utility function in equation (3), for the same target SIR the algorithm for which the target SIR is achieved with minimum power implies maximum utility function. We note that the values of the utility function implied by algorithm UTI1 are very close to those corresponding to the transmit powers yielded by the SOPC algorithm. We also note that the highest values of the utility function correspond to the transmit powers yielded by the EDPC algorithm, which suggests that the Nash equilibrium point achieved by algorithm UTI1 is inefficient. This observation agrees with [12] which shows analytically that the Nash equilibrium of the non-cooperative power control game implied by maximization of utility function in equation (3) is not efficient.

A similar plot containing the values of the utility function in 


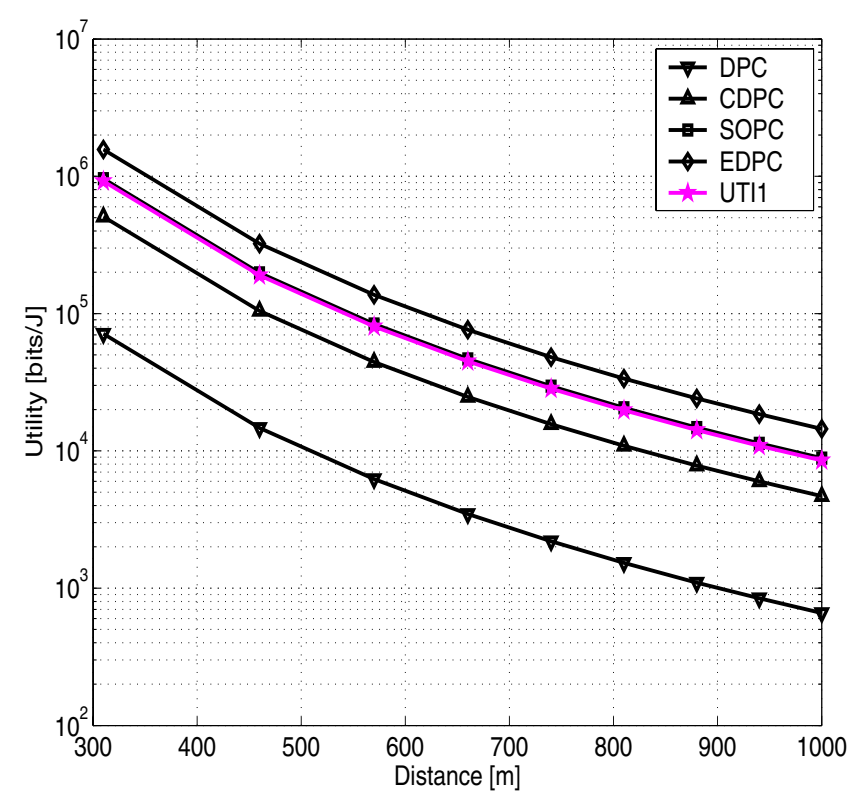

Fig. 2. Final user utilities computed using equation (3) for a system with 9 users after running various distributed power control algorithms discussed in section III.

equation (5) for transmit powers yielded by the DPC, CDPC, SOPC, and EDPC algorithms along with the value of the utility function yielded by algorithm UTI2 is given in Figure 3. As it can be noticed from the expression of the utility function in equation (5), in this case for uniform target/equilibrium SIRs and with pricing factors proportional to the path gains, users get the same utility. We note that the utilities implied by algorithm UTI2 are very close to those corresponding to the transmit powers yielded by the CDPC and SOPC algorithms. We also note that the highest values of the utility function correspond again to the transmit powers yielded by the EDPC algorithm, which suggests that the Nash equilibrium point achieved by algorithm UTI2 is also an inefficient one.

\section{CONCLUSIONS}

In this paper we investigate the use of power control algorithms established for cellular telephone systems in conjunction with utility functions used in wireless data systems. We compared these algorithms with power control algorithms based on game theory established relatively recently. Our analysis has shown that the CDPC and SOPC algorithms imply utilities similar to those achieved by the utility maximization algorithms UTI1 and UTI2. The analysis has also shown that the Nash equilibrium points to which the UTI1 and UTI2 algorithms converge are not efficient, and that better solutions are possible by using the EDPC algorithm. An analytical investigation of the EDPC algorithm in conjunction with utility functions will be the object of future research.

\section{ACKNOWLEDGMENTS}

This work was supported in part by the National Science Foundation under grant CCR-0312323.

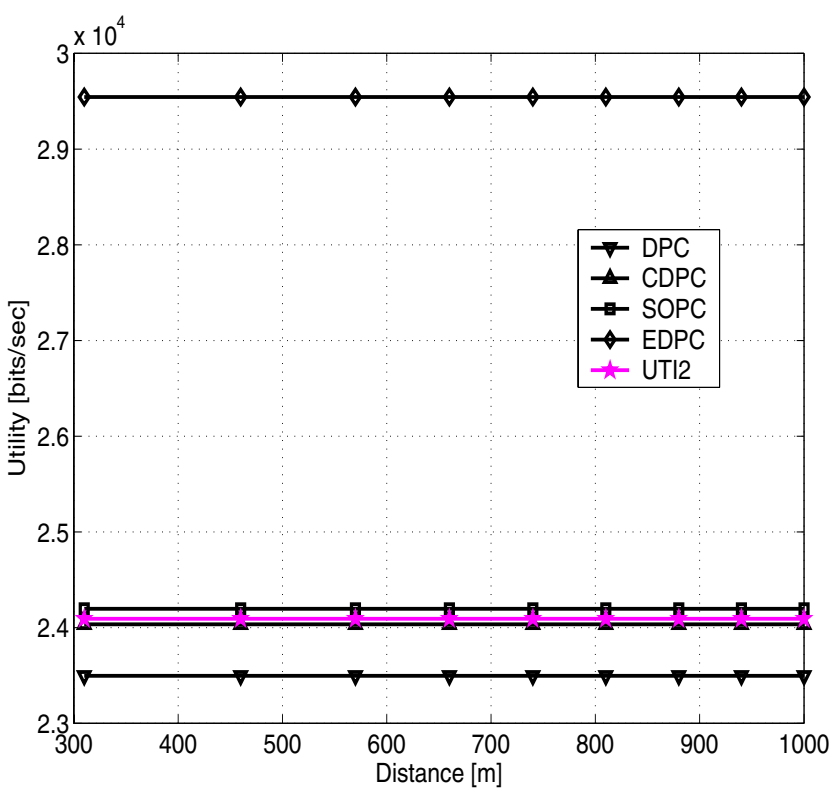

Fig. 3. Final user utilities computed using equation (5) for a system with 9 users after running various distributed power control algorithms discussed in section III.

\section{REFERENCES}

[1] N. Feng. Utility Maximization for Wireless Data Users Based on Power and Rate Control. Master's thesis, Rutgers University, Department of Electrical and Computer Engineering, 1999. Thesis Director: Prof. N. Mandayam.

[2] G. J. Foschini and Z. Miljanić. A Simple Distributed Autonomous Power Control Algorithm and its Convergence. IEEE Transactions on Vehicular Technology, 42(4):641-646, November 1993.

[3] D. J. Goodman and N. B. Mandayam. Power Control for Wireless Data. IEEE Personal Communications Magazine, 7(2):48-54, April 2000.

[4] S. A. Grandhi, R. Vijayan, and D. J. Goodman. Distributed Power Control in Cellular Radio Systems. IEEE Transactions on Communincations, 42(2/3/4):226-228, February/March/April 1994.

[5] S. A. Grandhi, R. Vijayan, D. J. Goodman, and J. Zander. Centralized Power Control in Cellular Radio Systems. IEEE Transactions on Vehicular Technology, 42(4):466-468, November 1993.

[6] S. A. Grandhi, R. Yates, and D. J. Goodman. Resource Allocation for Cellular Radio Systems. IEEE Transactions on Vehicular Technology, 46(3):581-587, August 1997.

[7] S. A. Grandhi and J. Zander. Constrained Power Control in Cellular Radio Systems. In Proceedings $44^{\text {th }}$ IEEE Vehicular Technology Conference - VTC'94, volume 2, pages 824-828, Stockholm, Sweden, June 1994.

[8] S. Gunturi and F. Paganini. A Game Theoretic Approach to Power Control in Cellular CDMA. In Proceedings $58^{\text {th }}$ IEEE Vehicular Technology Conference - VTC 2003 Fall, volume 3, pages 2362-2366, Orlando, FL, October 2003.

[9] R. Jäntti and S.-L. Kim. Second-Order Power Control with Asymptotically Fast Convergence. IEEE Journal on Selected Areas in Communications, 18(3):447-457, March 2000.

[10] R. D. Luce and H. Raiffa. Games and Decisions. John Wiley \& Sons, New York, NY, 1967

[11] L. Lv, S. Zhu, and S. Dong. Fast Convergence Distributed Power Control Algorithm for WCDMA Systems. IEE Proceedings on Communications, 150(2):134-140, April 2003.

[12] C. U. Saraydar, N. B. Mandayam, and D. J. Goodman. Efficient Power Control via Pricing in Wireless Data Networks. IEEE Transactions on Communications, 50(2):291-303, February 2002.

[13] R. Yates. A Framework for Uplink Power Control in Cellular Radio Systems. IEEE Journal on Selelected Areas in Communincations, 13(7):1341-1348, September 1995. 